\title{
Phase- and group-matched nonlinear interactions mediated by multiple filamentation in Kerr media
}

\author{
D. Faccio, ${ }^{1}$ A. Dubietis, ${ }^{2}$ G. Tamosauskas, ${ }^{2}$ P. Polesana, ${ }^{1,2}$ G. Valiulis, ${ }^{2}$ A. Piskarskas,,${ }^{2}$ A. Lotti, ${ }^{1}$ A. Couairon, ${ }^{3}$ and \\ P. Di Trapani ${ }^{1,2}$ \\ ${ }^{1}$ CNISM and Department of Physics and Mathematics, Università dell'Insubria, Via Valleggio 11, IT-22100 Como, Italy \\ ${ }^{2}$ Department of Quantum Electronics, Vilnius University, Sauletekio Ave. 9, bldg. 3, LT-10222, Vilnius, Lithuania \\ ${ }^{3}$ Centre de Physique Théorique, CNRS, École Polytechnique, F-91128, Palaiseau, France
}

(Received 30 July 2007; published 26 November 2007)

\begin{abstract}
Laser-pulse filamentation in Kerr media induced by a strongly elliptic input beam leads to breakup into a periodic array of filaments. In the plane of the filament array we observe strong Stokes and anti-Stokes emission at large angles. For 1 ps input pulses the measured angles are found to support two different phase-matching configurations: one nontransversally phase matched and the other perfectly phase matched. Shorter 200 fs pulses give rise to group-velocity-matched Raman conversion.
\end{abstract}

DOI: 10.1103/PhysRevA.76.055802

PACS number(s): 42.65.Re, 42.65.Jx, 42.65.Dr

High power ultrashort laser pulses propagating in nonlinear Kerr media undergo space-time self-focusing and form filaments, i.e., tightly localized in space $(10-100 \mu \mathrm{m})$ and in time (5-50 fs) intensity peaks surrounded by a much larger energy reservoir that coexist and propagate over long distances. This particular structure and the details of the filament dynamics may be interpreted in terms of conical [1] and, more generally, of nonlinear $\mathrm{X}$ waves [2,3]. Recently the filamentation regime has also been demonstrated to be extremely favorable for nonlinear interactions and frequency conversion through four wave mixing (FWM) [4] or stimulated Raman scattering (SRS) [5], thus suggesting possible useful outcomes of future applications in this field.

If the input power largely exceeds the critical power for self-focusing, instead of forming a single filament, the beam breaks up into multiple filaments (MF) [6]. This breakup usually exhibits complex propagation behavior and shows random shot-to-shot fluctuations, and is seeded by any noise present in the input beam $[7,8]$. In recent years, a progress toward a controlled beam breakup into multiple filaments has been achieved. The majority of the methods rely upon the modifications of the input beam parameters, e.g., amplitude and phase. Controlled MF patterns were generated by means of controlled phase aberrations $[9,10]$, and phase masks $[11]$, by introducing periodic amplitude modulation by the use of meshes and grids $[12,13]$, or by controlling the focusing conditions by means of a deformable mirror [14]. Another way toward controlled MF exploits launching an elliptical beam and has proved to be successful in overcoming random small-scale filamentation. Beam breakup starts evolving along the major axis and ends up with stable MF patterns. To this regard, reproducible MF patterns by slightly elliptical beams had been observed in various nonlinear media: water [15], air [16], BK7 glass [17], fused silica [18], and carbon disulfide [19], all revealing the universality of the deterministic breakup process. Moreover, recent studies have demonstrated that highly elliptical beams form one- or twodimensional arrays of regularly spaced filaments [20].

In this Brief Report we report the breakup of a highly input elliptical beam into a periodic filament array and the occurrence of nonlinear frequency coupling, mediated by phase-matched FWM and group-matched SRS, between the individual filaments. For input energies above the threshold for the formation of multiple filaments we observe redshifted and blueshifted signals that are emitted only in the plane of the filament array and at large angles with respect to the propagation direction. This emission is ascribed to the interplay between stimulated Raman scattering and four wave mixing. By changing the input pulse duration we are able to switch between a FWM-dominated process to a situation in which SRS alone is observed.

Experiments were carried out by launching 1 ps or $200 \mathrm{fs}$, $527 \mathrm{~nm}$ pulses at a repetition rate of $10 \mathrm{~Hz}$ provided by a Nd:glass laser system (Twinkle, Light Conversion Ltd., Vilnius, Lithuania). The beam was focused into a fused silica cell containing water using a cylindrical lens giving an elliptical with beam a minor axis of $100 \mu \mathrm{m}$ FWHM and ellipticity $e=12$. Figures 1 (a) -1 (c) show the near fields of $200 \mathrm{fs}$ pulses imaged onto a charge-coupled device (CCD) camera $(\mathrm{COHU})$ of the input beam (a) and of the MF array that forms for an input pump energy $E_{\text {pump }}=29 \mu \mathrm{J}$ at propagation distances of 2.4 (b) and $3.2 \mathrm{~cm}$ (c). The input beam breaks up into a periodic array of filaments characterized by a periodicity, for our experimental settings, $\Lambda \sim 75 \mu \mathrm{m}$ : similar features were observed with 1 ps pulses. Associated to the MFs, we noted a remarkably strong emission at redshifted and blueshifted (with respect to the pump) frequencies, both occurring at large propagation angles with respect to the propagation direction of the pump and only along the input beam ellipse major axis as can be seen in the spatial far-field pattern shown in Fig. 1(d). We thus measured the spectral farfield distributions $(\theta, \lambda)$ using an imaging spectrometer and a Nikon CCD camera, as described in detail elsewhere [21].

Figure 2(a) shows the measured $(\theta, \lambda)$ spectrum for $E_{\text {pump }}=100 \mu \mathrm{J}$ and input pulse duration of $1 \mathrm{ps}$. Around the pump wavelength a clear X-like structure is observed, thus indicating that recent findings and models based on the spontaneous formation and parametric interaction of $\mathrm{X}$ waves in single filaments remain valid also in the case of MFs [2,3]. The main difference with respect to the single filament case is that the $\mathrm{X}$ features visible around the pump wavelength are now somewhat broadened and hazier due to the superposi- 


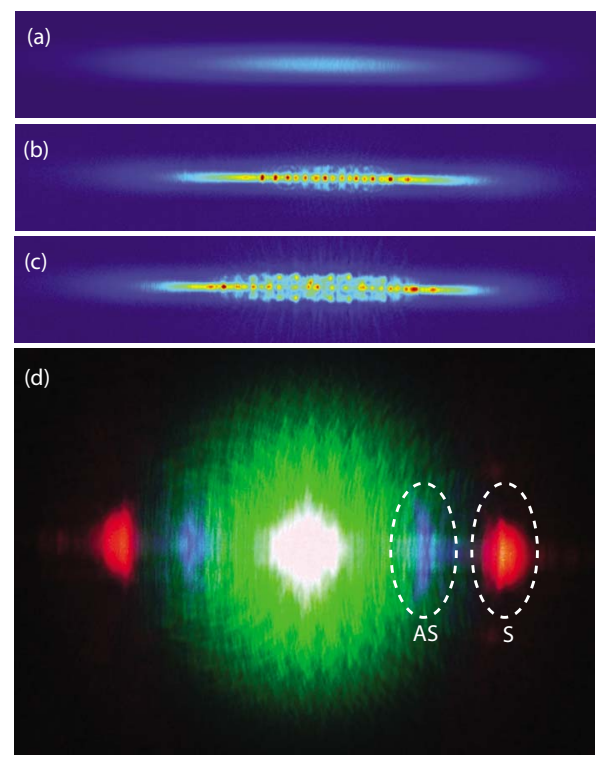

FIG. 1. (Color online) (a) Highly elliptic 200 fs input beam profile, (b) periodic array of filaments observed after $2.4 \mathrm{~cm}$ nonlinear propagation with $E_{\text {pump }}=29 \mu \mathrm{J}$, and (c) after $3.2 \mathrm{~cm}$ nonlinear propagation. Horizontal (vertical) dimension is $3 \mathrm{~mm}$ $(370 \mu \mathrm{m})$. (d) shows the spatial far-field pattern: AS and S indicate the anti-Stokes and Stokes components, respectively.

tion and interference of spectra coming from many individual filaments. The other remarkable feature is the intense signal observed around $\lambda=637 \mathrm{~nm}$ (both on and off axis) and $\lambda=450 \mathrm{~nm}$ (only off axis). These two wavelengths correspond precisely to the expected Stokes $(\mathrm{S})$ and anti-Stokes (AS) Raman shifts in water [22]. A weaker signal is also present at the second-order AS wavelength, $\lambda=391 \mathrm{~nm}$ and, due to a sharp drop in sensitivity in our CCD camera, we verified the presence of the second-order $\mathrm{S}$ component using a fiber-coupled spectrometer (Ocean Optics Inc., model QE65000). Thus it is clear that a strong interplay between SRS and FWM is occurring: due to the absence of this effect in the single filament regime it is also clear that this phenomenon is tightly linked to the formation of the MF array.

It has been pointed out that various phase-matching configurations may be proposed in order to explain some of the spectral features observed in optical filaments [22]. First of all we note that while the $\mathrm{S}$ signal is present both on axis $\left(\theta=0^{\circ}\right)$ and off axis $\left(\theta \sim \pm 2.57^{\circ}\right)$, the AS signal is present only off axis. Furthermore close inspection reveals that the (first-order) AS signal is split into two distinct angles, $\theta \sim \pm 1.73^{\circ}$ and $\theta \sim \pm 2.6^{\circ}$. We explain these features by assuming that two different FWM interactions are occurring, these being distinguished by their different phase-matching configurations. The first interaction is a longitudinally (nontransversally) phase-matched interaction between two onaxis pump photons, one on-axis $\mathrm{S}$ photon and an off-axis AS photon [as schematically shown in the inset marked with longitudinal phase matching (LPM) in Fig. 2(b)]. Phasematching conditions of this type are also fulfilled in single filaments: the high spatial localization of the central intensity peak allows a certain indetermination in the momentum conservation along the transverse direction [22]. Indeed it has

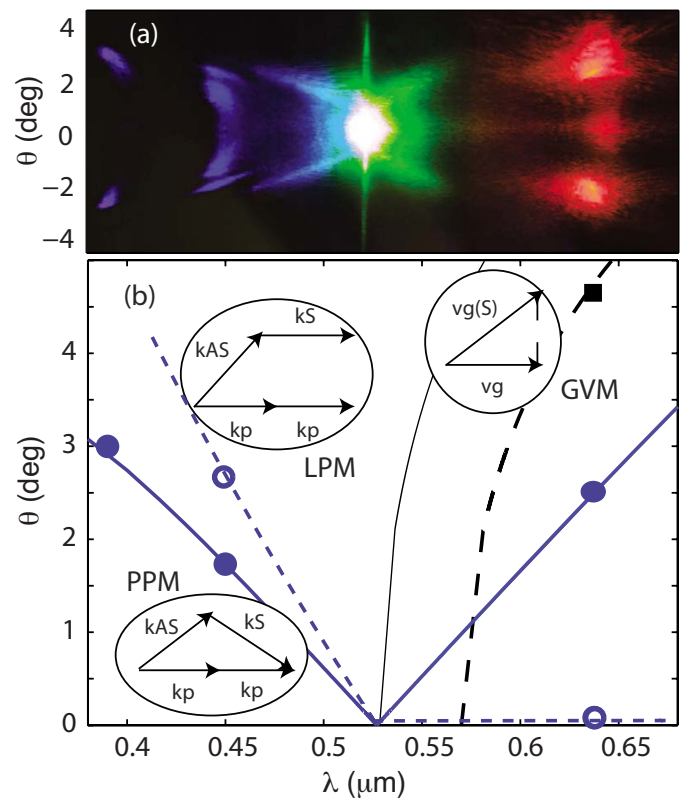

FIG. 2. (Color online) (a) Experimentally measured $(\theta, \lambda)$ spectrum of the multiple filaments with $E_{\text {pump }}=100 \mu \mathrm{J}$. (b) Summary of $(\theta, \lambda)$ coordinates at which emitted signals are observed. Shortdashed lines indicate longitudinal phase-matching curves (LPM), solid lines indicate the perfectly phase-matched curves (PPM), and the thin-solid and long-dashed lines indicate the group-matching curves, calculated for the input pump wavelength and for a faster traveling $\mathrm{X}$ wave (as described in the text), respectively. The insets indicate the relative phase (LPM and PPM) or group (GVM) matching diagrams.

been shown that precisely in the case of optical filaments full, i.e., both longitudinal and transverse, momentum conservation would lead to spectral features and dynamics that are not compatible with those effectively observed in experiments and numerical simulations [23]. Using the Sellmeier coefficients for water given by Eq. (12) in Ref. [24], it is possible to calculate the relative phase-matching curve, indicated with a short-dashed line in Fig. 2(b). The hollow circle represents the first-order AS component measured at the larger angle and lies very close to the calculated curve. The second phase-matched interaction is a perfectly, i.e., longitudinally and tranversally phase-matched FWM, as indicated schematically in the inset labeled as perfectly phase matched (PPM) in Fig. 2(b). The solid lines in Fig. 2(b) show the calculated phase-matching curves and the solid circles represent the measured angles. Here too, an excellent agreement is found. We note that this perfectly phase-matched interaction is strongly inhibited in single filaments due to the fact that both AS and S signals travel at large angles with respect to the pump and, due to spatial walk-off, will experience only a negligible gain. Conversely, in the MF regime, strong amplification may occur along the direction of the filament array: spatial walk-off is compensated by the periodically modulated field. This interaction also appears to be somewhat stronger than the longitudinally phase-matched interaction indicating the important role played by the filament array in the FWM process. Indeed, the proposed FWM scheme implies that AS signals generated or amplified by one filament 


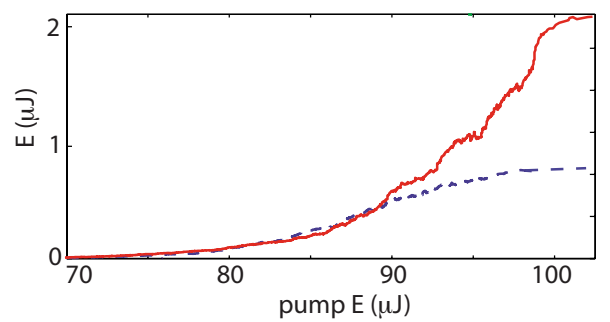

FIG. 3. (Color online) Measured Stokes (red solid line) and anti-Stokes (blue dashed line) energies for increasing input pump energy.

will interact nonlinearly with the S signal generated in neighboring (and possibly all other) filaments in the array.

The phase-matching curves shown in Fig. 2(b) imply that any frequency couples satisfying energy conservation may be amplified by the filament array. In this particular case only the Raman S and AS wavelengths are effectively amplified due to the fact that SRS provides a seed for the FWM process, without the need for any additional input seed pulse. In particular, we may conjecture that the perfectly phasematched FWM process builds up as a cascaded process: first a weak Stokes signal is generated on axis within one or more filaments in the array. This signal then seeds the longitudinally phase-matched FWM process that appears clearly in the MF regime due to the possibility for the AS signal to be amplified in passing through neighboring filaments in the array. As this signal increases some energy may be transferred to the relatively close (in angle) perfectly phasematched AS angle, effectively seeding the perfectly phasematched FWM process and giving rise to the off-axis $\mathrm{S}$ signal. We also measured the off-axis S- and AS-signal energies (Fig. 3) and found that for large input pump energies $(100 \mu \mathrm{J})$ the $\mathrm{S}$-signal energy $(\sim 2 \mu \mathrm{J})$ is notably larger than the AS energy $(\sim 0.9 \mu \mathrm{J})$. This indicates that although the conditions for phase matching are satisfied there is still a residual amplification due to SRS that does not require phase matching and that affects only the redshifted Stokes wavelength.

Finally, we repeated the experiment with a shorter 200 fs input pump pulse without varying any other parameters. We noted that all FWM processes were absent. Instead we measured a strong $\mathrm{S}$ signal, with no corresponding AS emission, at a large $4.8^{\circ}$ angle [solid square in Fig. 2(b)]. Due to the absence of the AS signal, we interpret the $S$ signal as the result of a group-velocity-matched (GVM) SRS process: by propagating at a specific angle, the longitudinal component of the Stokes pulse group velocity is equal to that of the on-axis pump. The thin solid line in Fig. 2(b) gives the $(\theta, \lambda)$ values that satisfy this condition assuming that the pump is traveling at the group velocity $v_{g}=2.211 \times 10^{8} \mathrm{~m} / \mathrm{s}$ of the input $527 \mathrm{~nm}$ Gaussian pulse. As can be seen this curve does not pass through the experimentally measured point. However, we should bear in mind that filamentation in condensed media is characterized by pulse splitting: one pulse will travel at a faster group velocity, the other at a slower group velocity with respect to the input pump. This splitting has been interpreted as an intrinsic feature of the spontaneous formation of $\mathrm{X}$ waves [3] and the specific group velocity of the two daughter pulses may be derived directly from the $(\theta, \lambda)$ spectra $[5,25,26]$. Performing this analysis (data not shown) the "fast" split pulse has $v_{g}=2.218 \times 10^{8} \mathrm{~m} / \mathrm{s}$ and we thus obtain the long-dashed curve in Fig. 2(b). This curve predicts very closely the measured Stokes emission angle. We note that the same group-matched SRS process is not allowed with the longer 1 ps input pump pulse due to the fact that the split pulses travel with significantly different group velocities with respect to the $200 \mathrm{fs}$ case. In particular, the spectrum in Fig. 2(a) gives $v_{g}=2.23 \times 10^{8} \mathrm{~m} / \mathrm{s}$ for the "fast" pulse. This velocity is larger than the Stokes group velocity $v_{g}=2.225 \times 10^{8} \mathrm{~m} / \mathrm{s}$ so that the matching of the longitudinal component of the S-pulse velocity with that of the pump cannot be fulfilled for any propagation angle.

In conclusion we have investigated spontaneous frequency conversion due to four wave mixing and stimulated Raman scattering mediated by multiple filament arrays. By changing the input pump pulse duration we are able to control the specific nonlinear process and therefore the characteristics of the frequency conversion: the long pulse dynamics are determined by FWM while those of short pulses are dominated by SRS alone. The investigated nonlinear interactions also clearly highlight the presence of a coupling mechanism and a channel for energy exchange between the individual filaments within the array.

The authors wish to acknowledge support from the Consorzio Nazionale Interunversitario per le Scienze della Materia (CNISM), progetto INNESCO, COFIN 2005, and access to research infrastructures activity in the Sixth Framework Programme of the EU (Contract No. RII3-CT-2003-506350, Laserlab Europe). P.P. acknowledges the support from Sixth EU Framework Programme Contract No. MEST-CF-2004008048 (ATLAS). P.D.T. acknowledges support from Marie Curie Chair project STELLA, Contract No. MEXC-CT2005-025710.
[1] A. Dubietis, E. Gaižauskas, G. Tamošauskas, and P. Di Trapani, Phys. Rev. Lett. 92, 253903 (2004).

[2] M. Kolesik, E. M. Wright, and J. V. Moloney, Phys. Rev. Lett. 92, 253901 (2004).

[3] D. Faccio, M. A. Porras, A. Dubietis, F. Bragheri, A. Couairon, and P. Di Trapani, Phys. Rev. Lett. 96, 193901 (2006).
[4] F. Théberge, N. Aközbek, W. Liu, A. Becker, and S. L. Chin, Phys. Rev. Lett. 97, 023904 (2006).

[5] D. Faccio, A. Averchi, A. Dubietis, P. Polesana, A. Piskarskas, P. Di Trapani, and A. Couairon, Opt. Lett. 32, 184 (2007).

[6] A. J. Campillo, S. L. Shapiro, and B. R. Suydam, Appl. Phys. Lett. 23, 628 (1973). 
[7] L. Berge et al., Phys. Rev. Lett. 92, 225002 (2004).

[8] M. Mlejnek, M. Kolesik, J. V. Moloney, and E. M. Wright, Phys. Rev. Lett. 83, 2938 (1999).

[9] G. Mèchain, A. Couairon, M. Franco, B. Prade, and A. Mysyrowicz, Phys. Rev. Lett. 93, 035003 (2004).

[10] G. Méchain, A. Couairon, Y. B. André, C. D’Amico, M. Franco, B. Prade, S. Tzortzakis, A. Mysyrowicz, and R. Sauerbrey, Appl. Phys. B: Lasers Opt. 79, 379 (2004).

[11] T. Pfeifer, L. Gallmann, M. J. Abel, D. M. Neumark, and S. R. Leone, Opt. Lett. 31, 2326 (2006).

[12] V. P. Kandidov, N. Aközbek, M. Scalora, O. G. Kosareva, A. V. Nyakk, Q. Luo, S. A. Hosseini, and S. L. Chin, Appl. Phys. B: Lasers Opt. 80, 267 (2005).

[13] H. Schroeder, J. Liu, and S. L. Chin, Opt. Express 12, 4768 (2004).

[14] Z. Jin, J. Zhang, M. H. Lu, Y. T. Li, Z. H. Wang, Z. Y. Wei, X. H. Yuan, and W. Yu, Opt. Express 13, 10424 (2005).

[15] A. Dubietis, G. Tamošauskas, G. Fibich, and B. Ilan, Opt. Lett. 29, 1126 (2004).

[16] G. Fibich, S. Eisenmann, B. Ilan, and A. Ziegler, Opt. Lett. 29, 1172 (2004).

[17] T. D. Grow and A. L. Gaeta, Opt. Express 13, 4594 (2005).
[18] V. Kudriašov, E. Gaižauskas, and V. Sirutkaitis, J. Opt. Soc. Am. B 22, 2619 (2005).

[19] M. Centurion, Y. Pu, and D. Psaltis, Opt. Express 13, 6202 (2005).

[20] A. Dubietis, E. Kučinskas, and G. Tamošauskas, Lith. J. Phys. 47, 27 (2007).

[21] D. Faccio, P. Di Trapani, S. Minardi, A. Bramati, F. Bragheri, C. Liberale, V. Degiorgio, A. Dubietis, and A. Matijosius, J. Opt. Soc. Am. B 22, 862 (2005).

[22] M. Wittmann and A. Penzkofer, Opt. Commun. 126, 308 (1996).

[23] D. Faccio, A. Averchi, A. Couairon, A. Dubietis, R. Piskarskas, A. Matijosius, F. Bragheri, M. A. Porras, A. Piskarskas, and P. Di Trapani, Phys. Rev. E 74, 047603 (2006).

[24] A. G. Van Engen, S. A. Diddams, and T. S. Clement, Appl. Opt. 37, 5679 (1998).

[25] M. Kolesik, E. M. Wright, and J. V. Moloney, Opt. Express 13, 10729 (2005).

[26] D. Faccio, A. Averchi, A. Couairon, M. Kolesik, J. V. Moloney, A. Dubietis, G. Tamosauskas, P. Polesana, A. Piskarskas, and P. Di Trapani, Opt. Express 15, 13077 (2007). 